Research Paper

\title{
Correlation between Candidate Single Nucleotide Variants and Several Clinicopathological Risk Factors Related to Breast Cancer in Jordanian Women: A Genotype-Phenotype Study
}

\author{
Laith N. AL-Eitan ${ }^{1,2 \bowtie}$, Doaa M. Rababa'h¹, Mansour A. Alghamdi ${ }^{3}$, Rame H. Khasawneh ${ }^{4}$ \\ 1. Department of Applied Biological Sciences, Jordan University of Science and Technology, Irbid 22110, Jordan \\ 2. Department of Biotechnology and Genetic Engineering, Jordan University of Science and Technology, Irbid 22110, Jordan \\ 3. College of Medicine, King Khalid University, Abha, Saudi Arabia \\ 4. Department of Hematopathology, King Hussein Medical Center (KHMC), Jordan Royal Medical Services (RMS), Amman 11118, Jordan \\ $\triangle$ Corresponding author: Dr. Laith N Al-Eitan at Jordan University of Science and Technology. P.O. Box 3030, Irbid 22110, Jordan. Tel: + (962) -2 -7201000. Ext. \\ 23462. Fax: + (962)-2-7201071. Email: Ineitan@just.edu.jo
}

(c) The author(s). This is an open access article distributed under the terms of the Creative Commons Attribution License (https://creativecommons.org/licenses/by/4.0/). See http:/ /ivyspring.com/terms for full terms and conditions.

Received: 2019.02.05; Accepted: 2019.06.12; Published: 2019.08.08

\begin{abstract}
This study aim to investigate the association of breast cancer risk and prognostic factors with single nucleotide variants of the BRCA1, BRCA2, DAPK1, MMP9, TOX3, and TP53 genes in Jordanian women. Blood samples were collected from 230 Jordanian breast cancer patients for use in DNA extraction followed by genotyping and subsequent statistical analysis. We found that two single nucleotide variants (SNVs) of the BRCA2 gene, namely rs1799944 and rs766173, were significantly associated with breastfeeding status. Likewise, the rs 11141901 and rs 1041326 SNVs of the DAPKI gene were linked with co-morbidity ( $p$-value $=0.002)$ and family history of $B C(p$-value $=0.015)$, while the rs $1045042 \mathrm{SNV}$ of the same gene was associated with both allergy ( $p$-value $=0.001)$ and family history of $B C(p$-value $=0.02)$. Tumor differentiation was correlated with the DAPKI SNVs rs 11141901 ( $p$-value $=0.041$ ) and rs 1041326 ( $p$-value $=0.005)$. Additionally, the rs2250889 SNV of the MMP9 gene was significantly associated with HER2 status, whereas the TP53 SNVs rs 12951053 and rs 1042522 were associated with age at menarche $(p$-value $=0.043)$ and breastfeeding status $(p$-value $=0.013)$, respectively. In contrast, the TP53 SNV rs2287497 was significantly linked to age at first pregnancy $(p$-value $=0.001)$, smoking $(p$-value $=0.041)$, and axillary lymph node status $\left(p\right.$-value $\left.=6 \mathrm{e}^{-4}\right)$. No such association was found for the BRCAl and TOX3 $\mathrm{SNV}$ s. The current findings suggest significant associations between certain SNVs and breast cancer risk and prognosis in Jordanian women.
\end{abstract}

\section{Background}

In recent years, the burden of disease in Jordan has shifted away from infectious illnesses and towards non-communicable diseases like cancer, the latter of which is responsible for $14 \%$ of all Jordanian deaths [1]. Cancer rates are exacerbated by the increased prevalence of risk factors for the disease as well as rising life expectancies among the Jordanian population [2]. Cancer risk factors can be broadly divided into those that are preventable, which are influenced by lifestyle and environment, and those that are unpreventable, such as age, family history, and individual genetic profiles [3]. Preventable risk factors that are notable among Jordanians are obesity and tobacco usage, each affecting nearly one-third of the population $[4,5]$. Similarly, non-preventable risk factors in the form of cancer-associated genetic mutations have been identified in Jordanians [6]. Despite this, genetic counselling remains under-utilized by the majority of the population, and the high rate of consanguineous unions in Jordanian 
society serves only to increase the risk of disease $[7,8]$.

In 2016 the incidence of breast cancer (BC) among Arab women was $(28 / 100,000)$ which is lower than the global mean $(46 / 100,000)$ [9]. Among Jordanian women, $(\mathrm{BC})$ is the most common type of cancer, accounting for two out of five female cancers and $12.5 \%$ of all deaths [10,11]. In Jordan; $37.3 \%$ [12] of all female cancers are diagnosed as BC, this percentage is similar to that in morocco $(34.3 \%)$, Tunisia (31.9\%), Algeria (37\%) [13], Egypt (38.8\%) [14], and Lebanon (38.2\%) [15] but higher than those in Libya (23.2\%) and Saudi Arabia (22.4\%) [13].

Reported risk factors for $\mathrm{BC}$ in Jordanians include postmenopausal obesity, breast trauma, irregular menstruation, and usage of fertility drugs, oral contraceptives, or hair dyes, while protective factors involve sufficient levels of physical activity and fruit/vegetable intake [16-18]. Mutations in $\mathrm{BC}$-associated genes have been investigated in Jordanian patients, and significant correlation has been found for certain single nucleotide variants (SNVs) of the BRCA1, BRCA2, DAPK1, MMP9, and TOX3 genes $[19,20]$.

It is imperative that guidelines for $\mathrm{BC}$ treatment be tailored for the Jordanian population, as the age of BC diagnosis for Arab women, including Jordanians, occurs almost a decade earlier than their Western counterparts [21].

Other than risk factors, prognostic factors encompassing a range of molecular subtyping and pathologic features play a major role in BC treatment outcome [22]. Molecular subtyping of BC utilizes estrogen, progesterone, and human epidermal growth factor receptor 2 (HER2) status to divide the disease into four subtypes: luminal A, luminal B, triple negative, and HER2-positive (Table 1) [23]. In Jordanian patients, the molecular subtype of $\mathrm{BC}$ differed depending on age, with $72 \%$ of women older than 50 and $42 \%$ of women younger than 50 having the luminal A subtype [24]. In another study of 752 Jordanian BC patients, triple negative BC was identified only in women under the age of 40 , while estrogen receptor expression was documented in the majority of cases [25]. Likewise, certain pathological features such as invasiveness, nodal involvement, tumor morphology, and tumor stage have been investigated in Jordanian BC patients, with the majority of cases being invasive carcinomas and tumor stage being the only statistically significant influence on 5-year survival rates $[25,26]$.

Despite the relatively high prevalence of the disease, rates of $\mathrm{BC}$ screening have been historically low in Jordan due to the cultural stigma surrounding cancer as well as socioeconomic inequalities [27,28]. In order to help alleviate this problem, it is important to understand how the preventable and non-preventable $\mathrm{BC}$ risk factors interplay to affect the disease's prognosis. Therefore, the primary objective of this study is to determine the extent of association between certain risk and prognostic factors for $\mathrm{BC}$ and previously reported $\mathrm{SNVs}$ in the cancer-associated BRCA1, BRCA2, DAPK1, MMP9, TOX3, and TP53 genes.

Table 1. Breast cancer (BC) classification according to molecular subtype

\begin{tabular}{llll}
$\begin{array}{l}\text { Molecular } \\
\text { subtype }\end{array}$ & $\begin{array}{l}\text { Estrogen receptor } \\
(\text { ER) }\end{array}$ & $\begin{array}{l}\text { Progesterone } \\
\text { receptor (PR) }\end{array}$ & HER2 \\
\hline Luminal A & ER(+) and /or PR(+) & & $(-)$ \\
Luminal B & ER(+) and /or PR(+) & & $(+)$ \\
Triple-negative & $(-)$ & $(-)$ & $(-)$ \\
HER+ & $(-)$ & $(-)$ & $(+)$ \\
\hline
\end{tabular}

\section{Materials and Methods}

\section{Experimental Design and Population}

Blood samples $(5 \mathrm{ml})$ were collected from 230 Jordanian BC patients recruited from the Jordanian Royal Medical Services Hospital in Amman, Jordan. All patients gave written informed consent prior to their participation, and this study was ethically approved by the Institutional Review Board (IRB) at Jordan University of Science and Technology.

\section{Selection of candidate Single Nucleotide Polymorphisms (SNPs)}

We selected a set of SNPs within candidate genes involved specifically in breast cancer from the PharmGKB database (http://www.pharmgkb.org) which provides an overview of significant polymorphisms involved in tumorgenesis processes. In addition, over than 60 genetic variants have been identified as predictable markers for breast cancer and the majority of them are involved in oncogenesis [29, 30]. The selected SNPs within BRCA2, DAPK1, MMP9, and TP53 genes were chosen through extensive review of a variety of sources and from reported polymorphisms associated with different types of cancers including breast cancer $[19,20,29,30]$.

\section{SNV Genotyping}

The Wizard ${ }^{\circledR}$ Genomic DNA Purification Kit (Promega Corp., USA) was used to extract genomic DNA from the blood samples. The integrity and concentration of the extracted DNA was then confirmed by agarose gel electrophoresis and the Nano-Drop ND-1000 UV-Vis Spectrophotometer (BioDrop, UK). $20 \mathrm{ng} / \mu \mathrm{l}$ samples were then shipped on wet ice to the Australian Genome Research Facility's (AGRF) Melbourne node for genotyping analysis via the Sequenom MassARRAY® system 
(iPLEX GOLD) (Sequenom, USA). The details of SNP genotyping and the description of study cohort were summarized in early published study by AL-Eitan et al (2017) [20].

\section{Statistical Analysis}

The $\chi^{2}$ test was performed to carry out the genotype-phenotype analyses in the present study using the Statistical Package for the Social Sciences (SPSS), version 25.0 (SPSS, Inc., Chicago, IL). The odds ratio (OR) was also calculated using binary logistic regression with 95\% confidence intervals (CI). P-values were considered to be significant if they were less than 0.05 .

\section{Results}

\section{Demographics of Experimental Population}

$74.6 \%$ of the 230 Jordanian BC patients were married with the average age $( \pm$ SD) being $53.19 \pm$ 12.777 years. $67.3 \%$ of the participants had breastfed at some point in their lives. In cross-sectional study, Khassawneh et al (2006) demonstrated that the initiation rate of breastfeeding of $88.6 \%$ which is higher than it reported in the USA for the same ages. However, it was less than breast feeding rates in other Middle Eastern countries [31]. While, the rate of exclusive breastfeeding (2013-2015) at 6 weeks among Jordanian mothers was $25.5 \%$ among, and this rate dropped to $2.1 \%$ at 6 months [32]. This decline in breastfeeding practicing among Jordanian women can be attributed to increased mothers' ages, employed mothers, and lack of breastfeeding education during pregnancy and after birth.

Likewise, the average ages of $\mathrm{BC}$ diagnosis and menopause were $50.90 \pm 12.2$ and $48.50 \pm 5.3$ years, respectively. Regarding co-morbidities, $36.3 \%$ of $\mathrm{BC}$ patients suffered from other complications such as hypertension, coronary artery disease, asthma, and diabetes mellitus. In terms of pathological BC features, $74.7 \%$ of all cases had been diagnosed with invasive ductal carcinoma compared to the $7.4 \%$ that were found to have in-situ ductal carcinoma. With regard to hormone receptor status, estrogen and progesterone receptors were found on the malignant cells of $77.5 \%$ and $68.8 \%$ of patients, respectively, while $35.4 \%$ of patients were positive for HER2 expression.

\section{Association of BRCA1 and BRCA2 SNVs with Prognostic and Risk Factors for BC}

None of the investigated BRCA1 SNVs showed any significant association with the risk factors for and pathological features of $\mathrm{BC}$ in Jordanian patients (Tables $3 \mathrm{a}$ and $3 \mathrm{~b}$ ). In contrast, the BRCA2 SNVs rs1799944 and rs766173 were both significantly associated with breastfeeding status, having p-values of 0.041 and 0.002 , respectively. However, like BRCA1, no BRCA2 SNV was found to be associated with pathological BC features in Jordanian patients (Tables $2 \mathrm{a}$ and $2 \mathrm{~b}$ ).

Table 2a. Association between different BRCAI and BRCA2 SNVs and risk factors for breast cancer (BC)

\begin{tabular}{|c|c|c|c|c|c|c|c|}
\hline \multirow[t]{2}{*}{ Risk factor } & \multicolumn{5}{|c|}{ BRCA1 } & \multicolumn{2}{|c|}{ BRCA2 } \\
\hline & rs8176318 & rs8176265 & rs3737559 & rs16940 & rs799905 & rs1799944 & rs766173 \\
\hline \multirow[t]{2}{*}{ Age at $\mathrm{BC}$ diagnosis ** } & $0.850^{\mathrm{a}}$ & $0.899 a$ & $0.532^{\mathrm{a}}$ & $0.781^{\mathrm{a}}$ & $0.196^{\mathrm{a}}$ & $0.733^{a}$ & $0.242^{\mathrm{a}}$ \\
\hline & $0.035^{b}$ & $0.016^{\mathrm{b}}$ & $0.390^{\mathrm{b}}$ & $0.08^{\mathrm{b}}$ & $1.672^{\mathrm{b}}$ & $0.116^{\mathrm{b}}$ & $1.369^{b}$ \\
\hline \multirow{2}{*}{$\underset{* *}{\text { Age at first pregnancy }}$} & $0.454^{\mathrm{a}}$ & $0.401^{\mathrm{a}}$ & $0.710^{\mathrm{a}}$ & $0.406^{\mathrm{a}}$ & $0.185^{\mathrm{a}}$ & $0.462^{\mathrm{a}}$ & $0.346^{\mathrm{a}}$ \\
\hline & $0.560^{\mathrm{b}}$ & $0.705^{\mathrm{b}}$ & $0.138^{\mathrm{b}}$ & $0.690^{\mathrm{b}}$ & $1.757^{b}$ & $0.541^{\mathrm{b}}$ & $0.888^{\mathrm{b}}$ \\
\hline \multirow[t]{2}{*}{ Age at menarche ** } & $0.971^{\mathrm{a}}$ & $0.931^{\mathrm{a}}$ & $0.589 \mathrm{a}$ & $0.651 \mathrm{a}$ & $0.762^{a}$ & $0.152^{\mathrm{a}}$ & $0.141^{\mathrm{a}}$ \\
\hline & $0.001^{b}$ & $0.007 \mathrm{~b}$ & $0.291^{b}$ & $0.204^{\mathrm{b}}$ & $0.091^{b}$ & $2.052^{b}$ & $2.167 \mathrm{~b}$ \\
\hline \multirow[t]{2}{*}{ Age at menopause ${ }^{* *}$} & $0.467 \mathrm{a}$ & $0.303^{a}$ & $0.330^{\mathrm{a}}$ & $0.302^{\mathrm{a}}$ & $0.301^{\mathrm{a}}$ & $0.763^{a}$ & $0.457 \mathrm{a}$ \\
\hline & $0.529 \mathrm{~b}$ & $1.06^{\mathrm{b}}$ & $0.948^{b}$ & $1.06^{\mathrm{b}}$ & $1.07 \mathrm{~b}$ & $0.09 \mathrm{~b}$ & $0.553^{\mathrm{b}}$ \\
\hline \multirow[t]{2}{*}{ Allergy * } & $0.985^{\mathrm{a}}$ & $0.881^{\mathrm{a}}$ & $0.401^{\mathrm{a}}$ & $0.989^{a}$ & $0.887^{a}$ & $0.347^{a}$ & $0.525^{\mathrm{a}}$ \\
\hline & $0.0003^{\mathrm{b}}$ & $0.022^{b}$ & $0.705^{b}$ & $0.0002^{b}$ & $0.020^{\mathrm{b}}$ & $0.884^{\mathrm{b}}$ & $0.404^{\mathrm{b}}$ \\
\hline \multirow[t]{2}{*}{ Breastfeeding status * } & $0.760^{\mathrm{a}}$ & $0.716^{\mathrm{a}}$ & $0.283^{a}$ & $0.608^{a}$ & $0.921^{\mathrm{a}}$ & $0.041^{\mathrm{a}}$ & $0.002^{\mathrm{a}}$ \\
\hline & $0.093^{b}$ & $0.132^{b}$ & $1.153^{b}$ & $0.263^{b}$ & $0.01^{\mathrm{b}}$ & $4.176^{b}$ & $9.55^{\mathrm{b}}$ \\
\hline \multirow[t]{2}{*}{ Body mass index ** } & $0.458^{a}$ & $0.391^{\mathrm{a}}$ & $0.489 \mathrm{a}$ & $0.223^{a}$ & $0.989 a$ & $0.453^{a}$ & $0.325^{\mathrm{a}}$ \\
\hline & $0.550^{\mathrm{b}}$ & $0.735^{b}$ & $0.478^{b}$ & $1.485^{\mathrm{b}}$ & $0.0002^{\mathrm{b}}$ & $0.563^{b}$ & $0.968^{\mathrm{b}}$ \\
\hline \multirow[t]{2}{*}{ Co-morbidity * } & $0.489 a$ & $0.780^{a}$ & $0.701^{\mathrm{a}}$ & $0.706^{\mathrm{a}}$ & $0.202^{a}$ & $0.214^{\mathrm{a}}$ & $0.147 \mathrm{a}$ \\
\hline & $0.478^{b}$ & $0.078^{b}$ & $0.147 \mathrm{~b}$ & $0.142^{\mathrm{b}}$ & $1.628 \mathrm{~b}$ & $1.544^{\mathrm{b}}$ & $2.10^{\mathrm{b}}$ \\
\hline \multirow[t]{2}{*}{ Family history * } & $0.499 \mathrm{a}$ & $0.423^{a}$ & $0.592^{\mathrm{a}}$ & $0.388^{\mathrm{a}}$ & $0.604^{a}$ & $486^{a}$ & $0.531^{\mathrm{a}}$ \\
\hline & $0.457 \mathrm{~b}$ & $0.642^{b}$ & $0.3^{b}$ & $0.745^{\mathrm{b}}$ & $0.269 \mathrm{~b}$ & $0.485^{\mathrm{b}}$ & $0.392^{b}$ \\
\hline \multirow[t]{2}{*}{ Smoking * } & $0.689^{a}$ & $0.671^{\mathrm{a}}$ & $0.287^{a}$ & $0.703^{a}$ & $0.914^{\mathrm{a}}$ & $0.125^{\mathrm{a}}$ & $0.165^{\mathrm{a}}$ \\
\hline & $0.16^{\mathrm{b}}$ & $0.180^{\mathrm{b}}$ & $1.13^{\mathrm{b}}$ & $0.145^{\mathrm{b}}$ & $0.012^{\mathrm{b}}$ & $2.354^{\mathrm{b}}$ & $1.928^{\mathrm{b}}$ \\
\hline
\end{tabular}

a: P-Value, b: Chi squared value. ${ }^{*}$ Pearson's chi-squared test was used to determine genotype-phenotype association. ${ }^{* *}$ Analysis of variance (ANOVA) was used to determine

genotype-phenotype association. P-Value $<0.05$ is considered as significant. 
Table 2b. Association between different BRCAl and BRCA2 SNVs and prognostic factors for breast cancer (BC)

\begin{tabular}{|c|c|c|c|c|c|c|c|}
\hline \multirow[t]{2}{*}{ Prognostic factor } & \multicolumn{5}{|c|}{ BRCA1 } & \multicolumn{2}{|c|}{$B R C A 2$} \\
\hline & rs8176318 & rs8176265 & rs3737559 & rs16940 & rs799905 & rs1799944 & rs766173 \\
\hline \multirow{2}{*}{ Axillary lymph nodes * } & $0.411^{\mathrm{a}}$ & $0.680^{\mathrm{a}}$ & $0.362^{\mathrm{a}}$ & $0.620^{\mathrm{a}}$ & $0.221^{\mathrm{a}}$ & $0.763^{a}$ & $0.659^{a}$ \\
\hline & $0.675^{b}$ & $1.883^{\mathrm{b}}$ & $0.830^{\mathrm{b}}$ & $0.245^{b}$ & $1.498 \mathrm{~b}$ & $0.090^{\mathrm{b}}$ & $0.194^{\mathrm{b}}$ \\
\hline \multirow{2}{*}{ Estrogen receptor status * } & $0.720 \mathrm{a}$ & $0.687 \mathrm{a}$ & $0.491^{a}$ & $0.675^{\mathrm{a}}$ & $0.798^{\mathrm{a}}$ & $0.902^{\mathrm{a}}$ & $0.868^{a}$ \\
\hline & $0.128^{b}$ & $0.162^{b}$ & $0.474^{\mathrm{b}}$ & $0.175^{b}$ & $0.065^{b}$ & $0.015^{b}$ & $0.027 \mathrm{~b}$ \\
\hline \multirow{2}{*}{ HER2 * } & $0.323 \mathrm{a}$ & $0.640^{\mathrm{a}}$ & $0.921^{\mathrm{a}}$ & $0.559 a$ & $0.449 a$ & $0.556^{\mathrm{a}}$ & $0.687 \mathrm{a}$ \\
\hline & $0.976^{\mathrm{b}}$ & $0.218^{\mathrm{b}}$ & $0.010^{\mathrm{b}}$ & $0.341^{b}$ & $0.596^{\mathrm{b}}$ & $0.346^{\mathrm{b}}$ & $0.162^{\mathrm{b}}$ \\
\hline \multirow{2}{*}{ In-situ vs. Invasive * } & $0.3801^{\mathrm{a}}$ & $0.683^{a}$ & $0.576^{\mathrm{a}}$ & $0.282^{\mathrm{a}}$ & $0.721^{\mathrm{a}}$ & $0.352^{a}$ & $0.341^{\mathrm{a}}$ \\
\hline & $0.770^{\mathrm{b}}$ & $0.166^{b}$ & $0.312^{\mathrm{b}}$ & $1.157 \mathrm{~b}$ & $0.127 \mathrm{~b}$ & $0.866^{b}$ & $0.906^{b}$ \\
\hline \multirow{2}{*}{ Lymph node involvement * } & $0.605^{\mathrm{a}}$ & $0.371^{\mathrm{a}}$ & $0.543^{a}$ & $0.276^{\mathrm{a}}$ & $0.432^{\mathrm{a}}$ & $0.582^{a}$ & $0.124^{\mathrm{a}}$ \\
\hline & $0.267 \mathrm{~b}$ & $0.800^{\mathrm{b}}$ & $0.37 \mathrm{~b}$ & $1.187 \mathrm{~b}$ & $0.617 \mathrm{~b}$ & $0.303^{b}$ & $2.366^{b}$ \\
\hline \multirow{2}{*}{ Progesterone receptor status * } & $0.765^{\mathrm{a}}$ & $0.688^{\mathrm{a}}$ & $0.621^{\mathrm{a}}$ & $0.735^{\mathrm{a}}$ & $0.786^{\mathrm{a}}$ & $0.403^{a}$ & $0.901^{\mathrm{a}}$ \\
\hline & $0.089 \mathrm{~b}$ & $0.161^{b}$ & $0.244^{b}$ & $0.114^{b}$ & $0.073^{b}$ & $0.699 \mathrm{~b}$ & $0.015^{\mathrm{b}}$ \\
\hline \multirow{2}{*}{ Tumor differentiation * } & $0.831^{\mathrm{a}}$ & $0.897 \mathrm{a}$ & $0.407 a$ & $0.942^{\mathrm{a}}$ & $0.385^{\mathrm{a}}$ & $0.879 a$ & $0.873^{a}$ \\
\hline & $0.045^{b}$ & $0.017 \mathrm{~b}$ & $0.687 \mathrm{~b}$ & $0.005^{b}$ & $0.755^{\mathrm{b}}$ & $0.023^{b}$ & $0.025^{\mathrm{b}}$ \\
\hline \multirow{2}{*}{ Tumor size ${ }^{* *}$} & $0.406^{\mathrm{a}}$ & $0.516^{\mathrm{a}}$ & $0.897 a$ & $0.521^{\mathrm{a}}$ & $0.453^{a}$ & $0.921^{\mathrm{a}}$ & $0.436^{a}$ \\
\hline & $1.803^{b}$ & $1.323^{b}$ & $0.217^{b}$ & $0.411^{b}$ & $1.584^{\mathrm{b}}$ & $0.01^{\mathrm{b}}$ & $1.66^{\mathrm{b}}$ \\
\hline \multirow{2}{*}{ Tumor stage * } & $0.510^{a}$ & $0.138^{a}$ & $0.084^{a}$ & $0.156^{\mathrm{a}}$ & $0.211^{\mathrm{a}}$ & $0.486^{a}$ & $0.706^{a}$ \\
\hline & $0.434^{b}$ & $2.2^{\mathrm{b}}$ & $2.986^{\mathrm{b}}$ & $2.013^{\mathrm{b}}$ & $1.565^{\mathrm{b}}$ & $0.485^{\mathrm{b}}$ & $0.142^{\mathrm{b}}$ \\
\hline
\end{tabular}

a: P-Value, b: Chi squared value. * Pearson's chi-squared test was used to determine genotype-phenotype association. ** Analysis of variance (ANOVA) was used to determine genotype-phenotype association. P-Value $<0.05$ is considered as significant.

Table 3a. Association between different DAPKI and MMP9 SNVs and risk factors for breast cancer $(B C)$

\begin{tabular}{lcccc}
\hline Risk factor & \multicolumn{2}{c}{ DAPK1 } & \multicolumn{2}{c}{ MMP9 } \\
\cline { 2 - 5 } & rs11141901 & rs1041326 & rs1045042 & rs2250889 \\
\hline Age at BC diagnosis & $0.987^{\mathrm{a}}$ & $0.263^{\mathrm{a}}$ & $0.503^{\mathrm{a}}$ & $0.488^{\mathrm{a}}$ \\
** & $0.0003^{\mathrm{b}}$ & $1.253^{\mathrm{b}}$ & $0.448^{\mathrm{b}}$ & $0.480^{\mathrm{b}}$ \\
Age at first pregnancy & $0.474^{\mathrm{a}}$ & $0.761^{\mathrm{a}}$ & $0.703^{\mathrm{a}}$ & $0.683^{\mathrm{a}}$ \\
** & $0.512^{\mathrm{b}}$ & $0.092^{\mathrm{b}}$ & $0.145^{\mathrm{b}}$ & $0.166^{\mathrm{b}}$ \\
Age at menarche ** & $0.392^{\mathrm{a}}$ & $0.935^{\mathrm{a}}$ & $0.315^{\mathrm{a}}$ & $0.681^{\mathrm{a}}$ \\
& $0.7327^{\mathrm{b}}$ & $0.006^{\mathrm{b}}$ & $1.01^{\mathrm{b}}$ & $0.169^{\mathrm{b}}$ \\
Age at menopause ** & $0.130^{\mathrm{a}}$ & $0.593^{\mathrm{a}}$ & $0.258^{\mathrm{a}}$ & $0.359^{\mathrm{a}}$ \\
& $2.29^{\mathrm{b}}$ & $0.285^{\mathrm{b}}$ & $1.279 \mathrm{~b}$ & $0.841^{\mathrm{b}}$ \\
Allergy * & $0.616^{\mathrm{a}}$ & $0.218^{\mathrm{a}}$ & $0.001^{\mathrm{a}}$ & $0.203^{\mathrm{a}}$ \\
& $0.251^{\mathrm{b}}$ & $1.517^{\mathrm{b}}$ & $10.83^{\mathrm{b}}$ & $1.621^{\mathrm{b}}$ \\
Breastfeeding status * & $0.488^{\mathrm{a}}$ & $0.738^{\mathrm{a}}$ & $0.139^{\mathrm{a}}$ & $0.871^{\mathrm{a}}$ \\
& $0.480^{\mathrm{b}}$ & $0.111^{\mathrm{b}}$ & $2.189^{\mathrm{b}}$ & $0.026^{\mathrm{b}}$ \\
Body mass index ** & $0.856^{\mathrm{a}}$ & $0.246^{\mathrm{a}}$ & $0.715^{\mathrm{a}}$ & $0.639^{\mathrm{a}}$ \\
& $0.032^{\mathrm{b}}$ & $1.346^{\mathrm{b}}$ & $0.133^{\mathrm{b}}$ & $0.220^{\mathrm{b}}$ \\
Co-morbidity * & $0.002^{\mathrm{a}}$ & $0.387^{\mathrm{a}}$ & $0.072^{\mathrm{a}}$ & $0.508^{\mathrm{a}}$ \\
Family history * & $9.55^{\mathrm{b}}$ & $0.748^{\mathrm{b}}$ & $3.237^{\mathrm{b}}$ & $0.438^{\mathrm{b}}$ \\
& $0.379^{\mathrm{a}}$ & $0.015^{\mathrm{a}}$ & $0.020^{\mathrm{a}}$ & $0.183^{\mathrm{a}}$ \\
Smoking * & $0.77^{\mathrm{b}}$ & $5.91^{\mathrm{b}}$ & $5.412^{\mathrm{b}}$ & $1.77^{\mathrm{b}}$ \\
& $0.373^{\mathrm{a}}$ & $0.684^{\mathrm{a}}$ & $0.884^{\mathrm{a}}$ & $0.214^{\mathrm{a}}$ \\
\hline
\end{tabular}

a: P-Value, b: Chi squared value. * Pearson's chi-squared test was used to determine genotype-phenotype association. ${ }^{* *}$ Analysis of variance (ANOVA) was used to determine genotype-phenotype association. P-Value $<0.05$ is considered as significant.

\section{Association of DAPK1 and MMP9 SNVs with Prognostic and Risk Factors for BC}

Tables $3 \mathrm{a}$ and $3 \mathrm{~b}$ illustrate the relationship between certain DAPK1 and MMP9 SNVs and clinical-pathologic BC features. The DAPK1 SNV rs11141901 showed a strong association with co-morbidity ( $p$-value $=0.002)$, while the rs1045042 SNV of the same gene was correlated with both allergy ( $p$-value $=0.001$ ) and family history of $\mathrm{BC}$ $(p$-value $=0.02)$. Family history of $\mathrm{BC}$ was also significantly associated with the DAPK1 SNV rs1041326 (p-value $=0.015)$. Concerning pathological features, tumor differentiation was significantly associated with the DAPK1 SNVs rs11141901 (p-value $=0.041$ ) and rs1041326 (p-value $=0.005$ ). For the investigated MMP9 SNVs, only rs2250889 showed an association with any clinical or pathological factor, namely HER2 status ( $p$-value $=0.044$ ).

\section{Association of TOX3 and TP53 SNVs with Prognostic and Risk Factors for BC}

The association between the investigated TOX3 and TP53 SNVs and certain BC features are shown in Tables $4 \mathrm{a}$ and $4 \mathrm{~b}$. The TOX3 SNV did not exhibit a significant relationship with any of the selected clinical and pathological BC features. In contrast, the TP53 SNVs rs12951053 and rs1042522 were found to be significantly associated with age at menarche $(p$-value $=0.043)$ and breastfeeding status $(p$-value $=$ 0.013), respectively. Additionally, the TP53 SNV rs2287497 was linked to both age at first pregnancy $(p$-value $=0.001)$ and smoking $(p$-value $=0.041)$. The only pathological feature to be linked to any TP53 SNV was axillary lymph node status, which was significantly associated with rs2287497 $\left(p\right.$-value $\left.=6 \mathrm{e}^{-4}\right)$.

Association of BRCA1, BRCA2, TP53, DAPK1, MMP9, and TOX3 SNVs with molecular subtype of $B C$

Despite the importance of molecular subtyping to $\mathrm{BC}$ prognosis and treatment, no association was found between these heterogenic markers and any candidate SNV in this study (Table 5).

\section{Discussion}

Breast cancer (BC) affects two out of five Jordanian women and is responsible for one out of ten deaths in Jordan $[10,11]$. Despite being a leading cause of morbidity and mortality, BC screening is not widespread among Jordanian women, and studies of the disease as it occurs in Jordanian women are not exhaustive $[2,27,28]$. The fact that $\mathrm{BC}$ risk and 
prognosis is modulated by ethnic differences demonstrates that research involving non-Jordanian women cannot simply be extrapolated to Jordanians, highlighting the need for studies involving Jordanian patients [33]. In the present study, the association of $\mathrm{BC}$ risk and prognosis with certain $\mathrm{SNVs}$ of the BRCA1, BRCA2, TP53, DAPK1, MMP9 and TOX3 genes was investigated.

Mutations within high-penetrance genes; breast cancer 1 (BRCA1) and breast cancer 2 (BRCA2) impact the underlying functions of those genes as tumor suppressor genes [34]. Moreover, BRCA1 and BRCA2 are responsible for DNA repair by homologous recombination. However, BRCA1/2 variants distributions are vary among populations, statistics about hereditary breast cancer among the Arabs origin are very scarce. For example, a Lebanese study was reported that $5.6 \%$ of high risk BC patients carried a deleterious BRCA1/2 mutations [35]. Another study was conducted in Moroccan women diagnosed with BC claimed that $31.6 \%$ of familial BC was found to be associated with BRCA1 mutations[36] while in Egypt $60 \%$ of familial BC cases were attributed to $B R C A 1$ mutations and approximately $26 \%$ were because of BRCA2 mutations [37]. A recent study in Jordan included 100 women diagnosed with $B C$ reported that $20 \%$ patients had deleterious $B R C A 1$ or BRCA2 mutations [19].

Table 3b. Association between different DAPK1 and MMP9 SNVs and prognostic factors for breast cancer (BC)

\begin{tabular}{|c|c|c|c|c|c|}
\hline \multirow[b]{2}{*}{ Prognostic factor } & \multicolumn{3}{|c|}{ DAPK1 } & \multicolumn{2}{|c|}{ MMP9 } \\
\hline & rs11141901 & rs1041326 & rs1045042 & rs2250889 & rs6065912 \\
\hline \multirow{2}{*}{ Axillary lymph nodes * } & $0.863^{a}$ & $0.401^{\mathrm{a}}$ & $0.586^{\mathrm{a}}$ & $0.645^{\mathrm{a}}$ & $0.565^{\mathrm{a}}$ \\
\hline & $0.029 \mathrm{~b}$ & $0.705^{b}$ & $0.296^{\mathrm{b}}$ & $0.212^{b}$ & $0.331^{b}$ \\
\hline \multirow{2}{*}{ Estrogen receptor status * } & $0.159 a$ & $0.598^{a}$ & $0.152^{\mathrm{a}}$ & $0.369 a$ & $0.631^{\mathrm{a}}$ \\
\hline & $1.984^{\mathrm{b}}$ & $0.278^{b}$ & $2.052^{b}$ & $0.807 \mathrm{~b}$ & $0.230^{\mathrm{b}}$ \\
\hline \multirow{2}{*}{ HER2 * } & $0.120^{\mathrm{a}}$ & $0.721^{\mathrm{a}}$ & $0.786^{\mathrm{a}}$ & $0.044^{\mathrm{a}}$ & $0.446^{\mathrm{a}}$ \\
\hline & $2.417 \mathrm{~b}$ & $0.127 \mathrm{~b}$ & $0.073^{b}$ & $4.05^{\mathrm{b}}$ & $0.580^{\mathrm{b}}$ \\
\hline \multirow{2}{*}{ In-situ vs. Invasive * } & $0.357 \mathrm{a}$ & $0.708^{a}$ & $0.704^{\mathrm{a}}$ & $0.540^{a}$ & $0.675^{\mathrm{a}}$ \\
\hline & $0.848^{\mathrm{b}}$ & $0.140^{\mathrm{b}}$ & $0.144^{b}$ & $0.375^{b}$ & $0.175^{\mathrm{b}}$ \\
\hline \multirow{2}{*}{ Lymph node involvement * } & $0.718^{\mathrm{a}}$ & $0.811^{\mathrm{a}}$ & $0.756^{\mathrm{a}}$ & $0.316^{\mathrm{a}}$ & $0.406^{\mathrm{a}}$ \\
\hline & $0.130^{\mathrm{b}}$ & $0.057 \mathrm{~b}$ & $0.096^{b}$ & $1.1^{\mathrm{b}}$ & $0.69 \mathrm{~b}$ \\
\hline \multirow{2}{*}{ Progesterone receptor status * } & $0.137 \mathrm{a}$ & $0.863^{a}$ & $0.571^{\mathrm{a}}$ & $0.086^{\mathrm{a}}$ & $0.663^{a}$ \\
\hline & $2.211^{\mathrm{b}}$ & $0.029 \mathrm{~b}$ & $0.321^{\mathrm{b}}$ & $2.94^{\mathrm{b}}$ & $0.189^{b}$ \\
\hline \multirow{2}{*}{ Tumor differentiation * } & $0.041^{\mathrm{a}}$ & $0.005^{a}$ & $0.631^{a}$ & $0.822^{a}$ & $0.401^{a}$ \\
\hline & $4.176^{\mathrm{b}}$ & $7.87 \mathrm{~b}$ & $0.230^{\mathrm{b}}$ & $0.05^{\mathrm{b}}$ & $0.71^{\mathrm{b}}$ \\
\hline \multirow{2}{*}{ Tumor size $* *$} & $0.982^{\mathrm{a}}$ & 0.368 & $0.318^{\mathrm{a}}$ & $0.469^{a}$ & $0.209^{a}$ \\
\hline & $0.036^{\mathrm{b}}$ & $1.99 \mathrm{~b}$ & $2.29 \mathrm{~b}$ & $1.51^{\mathrm{b}}$ & $3.131^{\mathrm{b}}$ \\
\hline \multirow{2}{*}{ Tumor stage * } & $0.442^{\mathrm{a}}$ & $0.608^{a}$ & $0.122^{\mathrm{a}}$ & $0.099 a$ & $0.805^{\mathrm{a}}$ \\
\hline & $0.591^{\mathrm{b}}$ & $0.275^{b}$ & $2.391^{\mathrm{b}}$ & $2.72^{\mathrm{b}}$ & $0.060^{\mathrm{b}}$ \\
\hline
\end{tabular}

a: P-Value, b: Chi squared value. * Pearson's chi-squared test was used to determine genotype-phenotype association. ** Analysis of variance (ANOVA) was used to determine genotype-phenotype association. P-Value $<0.05$ is considered as significant.

Table 4a. Association between different TOX3 and TP53 SNVs and risk factors for breast cancer (BC)

\begin{tabular}{|c|c|c|c|c|}
\hline \multirow[b]{2}{*}{ Risk factor } & \multirow{2}{*}{$\begin{array}{l}\text { TOX3 } \\
\text { rs1420546 }\end{array}$} & \multicolumn{3}{|l|}{ TP53 } \\
\hline & & rs12951053 & rs1042522 & rs2287497 \\
\hline \multirow{2}{*}{$\underset{* *}{\text { Age at BC diagnosis }}$} & $0.730^{a}$ & $0.116^{\mathrm{a}}$ & $0.546^{\mathrm{a}}$ & $0.814^{\mathrm{a}}$ \\
\hline & $0.119 \mathrm{~b}$ & $2.471^{\mathrm{b}}$ & $0.364^{\mathrm{b}}$ & $0.055^{\mathrm{b}}$ \\
\hline \multirow{2}{*}{$\underset{* *}{\text { Age at first pregnancy }}$} & $0.294^{a}$ & $0.751^{\mathrm{a}}$ & $0.267 \mathrm{a}$ & $0.001^{\mathrm{a}}$ \\
\hline & $1.101^{\mathrm{b}}$ & $0.101^{b}$ & $1.232^{\mathrm{b}}$ & $10.83^{b}$ \\
\hline \multirow{2}{*}{ Age at menarche $* *$} & $0.520 \mathrm{a}$ & $0.043^{a}$ & $0.423^{a}$ & $0.253^{a}$ \\
\hline & $0.413 b$ & $4.095 \mathrm{~b}$ & $0.642^{b}$ & $1.307^{b}$ \\
\hline \multirow{2}{*}{ Age at menopause ** } & $0.372^{\mathrm{a}}$ & $0.069 \mathrm{a}$ & $0.637 a$ & $0.189 \mathrm{a}$ \\
\hline & $0.797 \mathrm{~b}$ & $3.307 \mathrm{~b}$ & $0.222^{b}$ & $1.72^{\mathrm{b}}$ \\
\hline \multirow{2}{*}{ Allergy * } & $0.546^{\mathrm{a}}$ & $0.642^{a}$ & $0.323^{a}$ & $0.564^{a}$ \\
\hline & $0.364^{\mathrm{b}}$ & $0.216^{\mathrm{b}}$ & $0.976^{\mathrm{b}}$ & $0.332^{b}$ \\
\hline \multirow{2}{*}{ Breastfeeding status * } & $0.545^{\mathrm{a}}$ & $0.552^{\mathrm{a}}$ & $0.013^{\mathrm{a}}$ & $0.182^{\mathrm{a}}$ \\
\hline & $0.366^{\mathrm{b}}$ & $0.353^{\mathrm{b}}$ & $6.169 \mathrm{~b}$ & $1.781^{\mathrm{b}}$ \\
\hline \multirow{2}{*}{ Body mass index ** } & $0.155^{a}$ & $0.274^{a}$ & $0.587 \mathrm{a}$ & $0.568^{a}$ \\
\hline & $2.02^{b}$ & $1.197 \mathrm{~b}$ & $0.295^{b}$ & $0.326^{b}$ \\
\hline \multirow{2}{*}{ Co-morbidity * } & $0.375^{a}$ & $0.098^{a}$ & $0.953 a$ & $0.625^{a}$ \\
\hline & $0.787 \mathrm{~b}$ & $2.73^{b}$ & $0.003^{b}$ & $0.238^{b}$ \\
\hline \multirow{2}{*}{ Family history * } & $0.663^{a}$ & $0.788^{a}$ & $0.286^{\mathrm{a}}$ & $0.544^{a}$ \\
\hline & $0.189 \mathrm{~b}$ & $0.072^{\mathrm{b}}$ & $1.138^{\mathrm{b}}$ & $0.368^{b}$ \\
\hline \multirow{2}{*}{ Smoking * } & $0.366^{a}$ & $0.207 a$ & $0.078^{a}$ & $0.041^{\mathrm{a}}$ \\
\hline & $0.817 \mathrm{~b}$ & $1.59 \mathrm{~b}$ & $3.11^{b}$ & $4.17 \mathrm{~b}$ \\
\hline
\end{tabular}

Table 4b. Association between different TOX3 and TP53 SNVs and prognostic factors for breast cancer (BC)

\begin{tabular}{|c|c|c|c|c|}
\hline \multirow[b]{2}{*}{ Pathological BC features } & \multicolumn{4}{|c|}{ TOX3 TP53 } \\
\hline & rs1420546 & rs12951053 & rs1042522 & rs2287497 \\
\hline \multirow{2}{*}{ Axillary lymph nodes * } & $0.967 \mathrm{a}$ & $0.251^{\mathrm{a}}$ & $0.158^{a}$ & $6 e-4 a$ \\
\hline & $0.002^{b}$ & $1.318^{\mathrm{b}}$ & $1.993^{b}$ & $20.49 b$ \\
\hline \multirow{2}{*}{ Estrogen receptor status * } & $0.231^{\mathrm{a}}$ & $0.956^{\mathrm{a}}$ & $0.345^{\mathrm{a}}$ & $0.829 a$ \\
\hline & $1.435^{\mathrm{b}}$ & $0.003^{\mathrm{b}}$ & $0.891^{\mathrm{b}}$ & $0.046^{\mathrm{b}}$ \\
\hline \multirow{2}{*}{ HER2 * } & $0.152^{\mathrm{a}}$ & $0.366 a$ & $0.279 \mathrm{a}$ & $0.337 \mathrm{a}$ \\
\hline & $2.05^{b}$ & $0.817 \mathrm{~b}$ & $1.172^{\mathrm{b}}$ & $0.921^{b}$ \\
\hline \multirow{2}{*}{ In-situ vs. Invasive * } & $0.397 \mathrm{a}$ & $0.308^{a}$ & $0.736^{\mathrm{a}}$ & $0.589 a$ \\
\hline & $0.717 \mathrm{~b}$ & $1.04^{\mathrm{b}}$ & $0.113^{\mathrm{b}}$ & $0.291^{b}$ \\
\hline \multirow{2}{*}{ Lymph node involvement } & $0.589 \mathrm{a}$ & $0.629 \mathrm{a}$ & $0.419 \mathrm{a}$ & $0.631^{\mathrm{a}}$ \\
\hline & $0.291^{b}$ & $0.233^{b}$ & $0.653^{\mathrm{b}}$ & $0.230^{\mathrm{b}}$ \\
\hline \multirow{2}{*}{$\begin{array}{l}\text { Progesterone receptor } \\
\text { status* }\end{array}$} & $0.068^{a}$ & $0.469 a$ & $0.228^{a}$ & $0.946^{\mathrm{a}}$ \\
\hline & $3.33^{\mathrm{b}}$ & $0.524^{b}$ & $1.453^{b}$ & $0.004^{b}$ \\
\hline \multirow{2}{*}{ Tumor differentiation * } & $0.791^{\mathrm{a}}$ & $0.391^{\mathrm{a}}$ & $0.772^{\mathrm{a}}$ & $0.451^{\mathrm{a}}$ \\
\hline & $0.070^{\mathrm{b}}$ & $0.735^{\mathrm{b}}$ & $0.083^{b}$ & $0.56^{\mathrm{b}}$ \\
\hline \multirow{2}{*}{ Tumor size ** } & $0.489 a$ & $0.591^{\mathrm{a}}$ & $0.296^{\mathrm{a}}$ & $0.646^{\mathrm{a}}$ \\
\hline & $1.431^{\mathrm{b}}$ & $1.052^{\mathrm{b}}$ & $2.435^{\mathrm{b}}$ & $0.873^{b}$ \\
\hline \multirow{2}{*}{ Tumor stage * } & $0.892^{\mathrm{a}}$ & $0.261^{\mathrm{a}}$ & $0.381^{\mathrm{a}}$ & $0.297 \mathrm{a}$ \\
\hline & $0.018^{b}$ & $1.263 \mathrm{~b}$ & $0.767 \mathrm{~b}$ & $1.09 \mathrm{~b}$ \\
\hline
\end{tabular}
genotype-phenotype association. P-Value $<0.05$ is considered as significant. 
Table 5. Association between different intrinsic $B C$ subtypes and each BRCAI, BRCA2, TP53, DAPKI, MMP9, and TOX3 SNV

\begin{tabular}{|c|c|c|}
\hline Gene & SNV ID & $\begin{array}{c}\text { Luminal A vs Luminal B vs } \\
\text { Triple-negative }\end{array}$ \\
\hline \multirow{7}{*}{ BRCA1 } & \multirow{2}{*}{ rs16940 } & $0.442^{\mathrm{a}}$ \\
\hline & & $1.633^{\mathrm{b}}$ \\
\hline & \multirow{2}{*}{ rs799905 } & $0.823^{a}$ \\
\hline & & $0.389 \mathrm{~b}$ \\
\hline & \multirow{2}{*}{ rs8176318 } & $0.720^{a}$ \\
\hline & & $0.657 \mathrm{~b}$ \\
\hline & \multirow{2}{*}{ rs3737559 } & $0.701^{\mathrm{a}}$ \\
\hline & & $0.710^{\mathrm{b}}$ \\
\hline & rs8176265 & $0.766^{\mathrm{a}}$ \\
\hline \multirow{4}{*}{ BRCA2 } & \multirow{2}{*}{ rs1799944 } & $0.456 \mathrm{a}$ \\
\hline & & $1.571^{\mathrm{b}}$ \\
\hline & \multirow{2}{*}{ rs766173 } & $0.649 \mathrm{a}$ \\
\hline & & $0.864^{\mathrm{b}}$ \\
\hline \multirow{6}{*}{ DAPK1 } & \multirow{2}{*}{ rs11141901 } & $0.104^{a}$ \\
\hline & & $4.527^{\mathrm{b}}$ \\
\hline & \multirow{2}{*}{ rs1045042 } & $0.804^{a}$ \\
\hline & & $0.436^{\mathrm{b}}$ \\
\hline & \multirow{2}{*}{ rs1041326 } & $0.323^{\mathrm{a}}$ \\
\hline & & $2.26^{\mathrm{b}}$ \\
\hline \multirow{3}{*}{ MMP9 } & rs6065912 & $0.2733^{a}$ \\
\hline & \multirow{2}{*}{ rs2250889 } & $0.183 a$ \\
\hline & & $3.397 \mathrm{~b}$ \\
\hline \multirow{3}{*}{ TOX3 } & \multirow{2}{*}{ rs1420546 } & $0.065^{a}$ \\
\hline & & $5.467 \mathrm{~b}$ \\
\hline & rs1042522 & $0.093 \mathrm{a}$ \\
\hline \multirow{4}{*}{ TP53 } & \multirow{2}{*}{ rs12951053 } & $0.527 \mathrm{a}$ \\
\hline & & $1.281^{\mathrm{b}}$ \\
\hline & \multirow{2}{*}{ rs2287497 } & $0.212^{a}$ \\
\hline & & $3.102^{\mathrm{b}}$ \\
\hline
\end{tabular}

a: P-Value, b: Chi squared value. P-Value $<0.05$ is considered as significant.

The tumor protein 53 (TP53) gene is another high-penetrance breast cancer susceptibility gene. Mutation locus (upstream and downstream) in TP53 can influence gene function. rs1042522 SNP of TP53 has been suggested that is considered as prognostic marker associated with a low tumor grade in breast cancer [38]. Another gene suggested to be involved in breast cancer is death-associated protein kinase 1 (DAPK1) which is known in its role of inducing cell death and recognized as a tumor suppressor gene. Mutations within DAPK1 gene have implicated in down regulation of DAPK1 transcription [39].

Matrix Metallopeptidase 9 (MMP9) is a member of the Matrix Metalloproteinases (MMPs) family and considers as metastasis-associated gene. This gene is also involved in tumor growth, invasion, carcinogenesis and angiogenesis and has been found that the activity and the expression levels increase in malignant breast tumors. These make the MMP9 as a useful marker for BC prognosis [40]. While, The TOX high-mobility group box family member 3 (TOX3) is classified as low-penetrance gene that plays a crucial function in chromatin structure alteration. It has been also suggested that an amplified expression of TOX3 can lead to bone metastasis [41].

Mutations in the BRCA1 and BRCA2 genes are perhaps the most well-known causes of hereditary BC due to their disruption of the genes' tumorsuppressing functions [34]. Among Jordanian patients with a history of $\mathrm{BC}$, most screened mutations were found within exon 11 of the BRCA1 gene [42]. In another study, $20 \%$ of Jordanian $B C$ cases possessed deleterious mutations in the BRCA1 and BRCA2 genes, and the highest mutation rate was found in triple-negative tumors [19]. Our findings show that the BRCA1 SNVs rs8176318, rs8176265 rs3737559, rs16940, and rs799905 did not have any significant association with $\mathrm{BC}$ risk and prognosis in Jordanians. On the other hand, breastfeeding status was significantly associated with both the BRCA2 SNVs rs1799944 and rs766173.

Like the BRCA1 and BRCA2 genes, the death-associated protein kinase 1 (DAPK1) gene possesses tumor-suppressive properties, and mutations in this gene are particular associated with triple-negative BC [43]. In a previous study, the DAPK1 SNV rs11141901 was found to be significantly linked to increased BC risk in Jordanians [20]. The findings of the current study show that the DAPK1 SNV rs1045042 is significantly associated with allergy and family history of $\mathrm{BC}$, while the DAPK1 SNV rs1041326 is correlated with family history of BC and tumor differentiation. In addition, the DAPK1 SNV rs11141901 was significantly associated with both co-morbidity and tumor differentiation. On a similar note, the matrix metallopeptidase 9 (MMP9) gene has also been implicated in triple-negative $\mathrm{BC}$ risk and development due to its angiogenic and matrix remodeling functions [44]. It has been reported that the MMP9SNV rs6065912 was significantly associated with increased BC risk in Jordanians [20]. In contrast, our findings show that the MMP9 SNV rs2250889 was only significantly associated with HER2 status.

Likewise, the tumor protein p53 (TP53) gene has long been known its multitude of anticancer functions and mechanisms, and mutations in this gene have been reported to increase the metabolic capacity of BC cells [45]. Our findings show that the TP53 SNVs rs12951053 and rs1042522 were significantly associated with age at menarche and breastfeeding status, respectively, while the TP53 SNV rs2287497 was linked to age at first pregnancy, smoking, and axillary lymph node status. In contrast to the TP53 gene, the TOX high mobility group box family member 3 (TOX3) gene, which is involved in calcium-dependent transcription, has only recently been implicated in BC development [46]. In Jordanians, the TOX3 SNV rs1420546 was found to be associated with increased BC risk by a prior study [26]. Contrastingly, the present study did not demonstrate significant association between the 
TOX3 SNV rs1420546 and any factors related to BC risk and prognosis.

Molecular subtyping of BC differentiates between high-risk and low-risk patients, improving overall prognosis and therapeutic outcome [47]. In a study of 193 Jordanian patients, the most common BC subtype was found to be luminal A [34]. In contrast, the present findings do not show any significant association between the investigated $B R C A 1, B R C A 2$, DAPK1, MMP9, TOX3, and TP53 SNVs and molecular subtype of $B C$. However, more research needs to be carried out regarding $B C$ subtypes in Jordanians due to the fact that each subtype is associated with specific age-distribution patterns [48].

Conclusively, the current findings illustrate the relationship between specific BRCA2, DAPK1, MMP9, and TP53 SNVs and BC risk and prognosis in Jordanian women. Our findings shed some light on the nature of $\mathrm{BC}$ as it occurs among Jordanian women and could be used in awareness and prevention initiatives. Moreover, identifying prognostic factors that can predict the risk of cancer development and progression is an urgent need in clinical practice. Determining variants involved in breast cancer patients' prognosis can also help in stratifying patients in clinical trials and lead to identifying the most effective therapy to provide patients with personalized medicine treatment. Limitations of the present study include the relatively small sample size and the lack of data related to healthy controls. Future lines of research should incorporate a larger sample cohort comprising both cases and controls to understand the association of different genes with $\mathrm{BC}$ risk and prognosis.

\section{Abbreviations}

AGRF: Australian Genome Research Facility; BC: Breast Cancer; $X^{2}$ : Chi squared value; DNA: Deoxyribonucleic acid; ER: Etrogen Receptor; GWAS: genome-wide association study; GJRMS: Jordanian Royal Medical Services; HWE: Hardy-Weinberg equilibrium; Het: Heterozygote; Hz: Homozygote; HER2: Human epidermal growth factor receptor 2 marker; IRB: Institutional Review Board; PR: Progesterone Receptor; SNVs: Single nucleotide variants; SPSS: Statistical Package for the Social Sciences.

\section{Acknowledgements}

The authors thank the Jordanian Royal Medical Services (JRMS), Amman, Jordan, for approving this study in the first instance and making the clinical data and samples available for the study. This study was funded by the Deanship of Research (RN: 20140204), Jordan University of Science and Technology.

\section{Ethical approval and Informed consent}

All procedures performed in studies involving human participants were in accordance with the ethical standards of the Institutional Review Board (IRB) at Jordan University of Science and Technology with ethical code number (14/78/2014). Informed consent was obtained from all individual participants included in the study. Informed consent was obtained from all individual participants included in the study.

\section{Competing Interests}

The authors have declared that no competing interest exists.

\section{References}

[1] Al-Nsour M, Zindah M, Belbeisi A, Hadaddin R, Brown DW, Walke $\mathrm{H}$. Prevalence of selected chronic, noncommunicable disease risk factors in Jordan: results of the 2007 Jordan Behavioral Risk Factor Surveillance Survey. Prev Chronic Dis. 2012;9:E25.

[2] Abdel-Razeq H, Attiga F, Mansour A. Cancer care in Jordan. Hematol Oncol Stem Cell Ther. 2015;8:64-70. doi:10.1016/J.HEMONC.2015.02.001.

[3] Hayes J, Richardson A, Frampton C. Population attributable risks for modifiable lifestyle factors and breast cancer in New Zealand women. Intern Med J. 2013;43:1198-204. doi:10.1111/imj.12256.

[4] Khader Y, Batieha A, Ajlouni H, El-Khateeb M, Ajlouni K. Obesity in Jordan: prevalence, associated factors, comorbidities, and change in prevalence over ten years. Metab Syndr Relat Disord. 2008;6:113-20. doi:10.1089/met.2007.0030.

[5] Abughosh S, Wu I-H, Hawari F, Peters RJ, Yang M, Crutchley R, et al. Cigarette Smoking Among Jordanian Adults. J Ethn Subst Abuse. 2012;11:101-12. doi:10.1080/15332640.2012.674888.

[6] Dasouki M, El-Shanti H. Genetic Disorders in Jordan. Genet. Disord. Among Arab Popul., Berlin, Heidelberg: Springer Berlin Heidelberg. 2010, p. 325-52. doi:10.1007/978-3-642-05080-0_11.

[7] Ahram M, Soubani M, Abu Salem L, Saker H, Ahmad M. Knowledge, Attitudes, and Practice Regarding Genetic Testing and Genetic Counselors in Jordan: A Population-Based Survey. J Genet Couns. 2015;24:1001-10. doi:10.1007/s10897-015-9839-3.

[8] Hamamy HA, Masri AT, Al-Hadidy AM, Ajlouni KM. Consanguinity and genetic disorders. Profile from Jordan. Saudi Med J. 2007;28:1015-7.

[9] Chouchane L, Boussen H, Sastry K.Breast cancer in Arab populations: molecular characteristics and disease management implications. Lancet Oncol. 2013; 14(10):417-24.

[10] Qatamish N, Nusairat T. Think Pink - Jordan Breast Cancer Program (JBCP) Community Mobilization Initiative. J Glob Oncol. 2018:18s-18s. doi:10.1200/jgo.18.10400.

[11] Al-Hussami M, Zeilani $\mathrm{R}$, AlKhawaldeh OA, Abushaika L. Jordanian women's personal practices regarding prevention and early detection of breast cancer. Int J Nurs Knowl. 2014;25:189-94. doi:10.1111/2047-3095.12045.

[12] Abdel-Razeq H, Attiga F, Mansour A. Cancer care in Jordan. Hematol Oncol Stem Cell Ther. 2015;8:64-70. doi:10.1016/J.HEMONC.2015.02.001.

[13] Khalis M, El Rhazi K, Charaka H, et al. Female Breast Cancer Incidence and Mortality in Morocco: Comparison with Other Countries. Asian Pac J Cancer Prev. 2016;17(12):5211-5216. Published . doi:10.22034/APJCP.2016.17.12.5211

[14] Ibrahim A, Khaled H, Mikhail N, Baraka H, Kamel H. Cancer Incidence in Egypt: Results of the National Population-Based Cancer Registry Program. J Cancer Epidemiol. 2014; 1-8.

[15] Lakkis N, Adib S, Osman M, Musharafieh U, Hamadeh G. Breast cancer in Lebanon: Incidence and comparison to regional and Western countries. Cancer Epidemiol. 2010; 34: 221-22

[16] Atoum MF, Al-Hourani HM. Lifestyle related risk factors for breast cancer in Jordanian females. Saudi Med J. 2004;25:1245-8.

[17] Al Qadire M, Alkhalaileh M, Hina H. Risk Factors for Breast Cancer among Jordanian Women: A Case-control Study. Iran J Public Health. 2018:47:49-56.

[18] Petro-Nustas W, Norton ME, Al-Masarweh I. Risk Factors for Breast Cancer in Jordanian Women. J Nurs Scholarsh . 2002;34:19-25. doi:10.1111/j.1547-5069.2002.00019.x.

[19] Abdel-Razeq H, Al-Omari A, Zahran F, Arun B. Germline BRCA1/BRCA2 mutations among high risk breast cancer patients in Jordan. BMC Cancer. 2018;18:152. doi:10.1186/s12885-018-4079-1.

[20] AL-Eitan LN, Jamous RI, Khasawneh RH. Candidate Gene Analysis of Breast Cancer in the Jordanian Population of Arab Descent: A Case-Control Study. Cancer Invest. 2017;35:256-70. doi:10.1080/07357907.2017.1289217.

[21] Najjar H, Easson A. Age at diagnosis of breast cancer in Arab nations. Int J Surg. 2010;8:448-52. doi:10.1016/J.IJSU.2010.05.012. 
[22] Stankov A, Bargallo-Rocha JE, Silvio AÑ-S, Ramirez MT, Stankova-Ninova K, Meneses-Garcia A. Prognostic factors and recurrence in breast cancer: experience at the national cancer institute of Mexico. ISRN Oncol. 2012;2012:825258. doi:10.5402/2012/825258.

[23] Sadek RF, Zhang L fang, Abdul Sater HT. Breast cancer molecular subtypes association with clinical outcomes and race. J Clin Oncol. 2017;35:e12575-e12575. doi:10.1200/JCO.2017.35.15_suppl.e12575.

[24] Shomaf M, Masad J, Najjar S, Faydi D. Distribution of breast cancer subtypes among Jordanian women and correlation with histopathological grade: molecular subclassification study. JRSM Short Rep. 2013;4:2042533313490516. doi:10.1177/2042533313490516.

[25] Obeidat F, Ahram M, Al Khader A, Battah K, Alchalabi M, Melhem JM, et al. Clinical and histopathological features of breast cancer in Jordan: Experience from a tertiary care hospital. J Pak Med Assoc. 2017;67:1206-12.

[26] Arkoob K, Al-Nsour M, Al-Nemry O, Al-Hajawi B. Epidemiology of breast cancer in women in Jordan: patient characteristics and survival analysis. East Mediterr Health J. 2010;16:1032-8.

[27] Al Rifai R, Nakamura K. Differences in Breast and Cervical Cancer Screening Rates in Jordan among Women from Different Socioeconomic Strata: Analysis of the 2012 Population-Based Household Survey. Asian Pac J Cancer Prev. 2015;16:6697-704.

[28] Abu-Helalah MA, Alshraideh HA, Al-Serhan A-AA, Kawaleet M, Nesheiwat AI. Knowledge, barriers and attitudes towards breast cancer mammography screening in jordan. Asian Pac J Cancer Prev. 2015;16:3981-90.

[29] Bayraktar S, Thompson PA, Yoo S-Y, Do K-A, Sahin AA, Arun BK, et al. The relationship between eight GWAS-identified single-nucleotide polymorphisms and primary breast cancer outcomes. Oncologist. 2013;18: 493-500. 35.

[30] Pirie A, Guo Q, Kraft P, Canisius S, Eccles DM, Rahman N, et al. Common germline polymorphisms associated with breast cancer-specific survival. Breast Cancer Res. 2015;17:58.

[31] Amarin Z, Alkafajei A. Knowledge, attitude and practice of breastfeeding in the north of Jordan: a cross-sectional study. Int Breastfeed J. 2006;1:17.

[32] Al dasoqi K, Safadi R, Badran E, Basha AS, Jordan S, Ahmad M. Initiation and continuation of breastfeeding among Jordanian first-time mothers: a prospective cohort study. International Journal of Women's Health. 2018; 10: $571-577$

[33] Maskarinec G, Sen C, Koga K, Conroy SM. Ethnic differences in breast cancer survival: status and determinants. Womens Health (Lond Engl). 2011;7:677-87. doi:10.2217/whe.11.67.

[34] Roy R, Chun J, Powell SN. BRCA1 and BRCA2: different roles in a common pathway of genome protection. Nat Rev Cancer. 2011;12:68-78. doi:10.1038/nrc3181.

[35] Jalkh N, Nassar-Slaba J, Chouery E, Salem N, Uhrchammer N, Golmard L, et al. Prevalance of BRCA1 and BRCA2 mutations in familial breast cancer patients in Lebanon. Hered Cancer Clin Pract. 2012;10:7

[36] Laraqui A, Uhrhammer N, Lahlou-Amine I, EL Rhaffouli H, El Baghdadi J, Dehayni M, et al. Mutation screening of the BRCA1 gene in early onset and familial breast/ovarian cancer in Moroccan population. Int J Med Sci. 2013; 10:60-7.

[37] Ibrahim SS, Hafez EE, Hashishe MM. Presymptomatic breast cancer in Egypt: role of BRCA1 and BRCA2 tumor suppressor genes mutations detection. J Exp Clin Cancer Res. 2010;29:82-91.

[38] Dunning A, Healey C, Pharoah P, Teare M, Ponder B, Easton D. A systematic review of genetic polymorphisms and breast cancer risk. Cancer Epidemiol Biomarkers Prev. 1999;8:843-854

[39] Raval A, Tanner S, Byrd J, Angerman E, Perko J, Chen S, et al. Downregulation of death-associated protein kinase 1 (DAPK1) in chronic lymphocytic leukemia. Cell. 2007;129:879-890.

[40] Grieu F, Li W, Iacopetta B. Genetic polymorphisms in the MMP-2 and MMP9 genes and breast cancer phenotype. Breast Cancer Res Treat. 2004;88:197-204.

[41] Smid M, Wang Y, Klijn I, Sieuwerts A, Zhang Y, Atkins D, et al. Genes associated with breast cancer metastatic to bone. J Clin Oncol. 2006;24:2261-2267

[42] Atoum MF, Al-Kayed SA. Mutation analysis of the breast cancer gene BRCA1 among breast cancer Jordanian females. Saudi Med J. 2004;25:60-3.

[43] Zhao J, Zhao D, Poage GM, Mazumdar A, Zhang Y, Hill JL, et al. Death-associated protein kinase 1 promotes growth of p53-mutant cancers. J Clin Invest 2015;125:2707-20. doi:10.1172/JCI70805

[44] Mehner C, Hockla A, Miller E, Ran S, Radisky DC, Radisky ES. Tumor cell-produced matrix metalloproteinase 9 (MMP-9) drives malignant progression and metastasis of basal-like triple negative breast cancer. Oncotarget 2014;5:2736-49. doi:10.18632/oncotarget.1932.

[45] Harami-Papp H, Pongor LS, Munkácsy G, Horváth G, Nagy ÁM, Ambrus A, et al. TP53 mutation hits energy metabolism and increases glycolysis in breast cancer. Oncotarget 2016;7:67183-95. doi:10.18632/oncotarget.11594.

[46] Jones JO, Chin S-F, Wong-Taylor L-A, Leaford D, Ponder BAJ, Caldas C, et al. TOX3 mutations in breast cancer. PLoS One 2013;8:e74102. doi:10.1371/journal.pone.0074102.

[47] Rossing M, Østrup O, Majewski WW, Kinalis S, Jensen M-B, Knoop A, et al. Molecular subtyping of breast cancer improves identification of both high and low risk patients. Acta Oncol (Madr) 2018;57:58-66. doi:10.1080/0284186X.2017.1398416

[48] Anderson WF, Pfeiffer RM, Dores GM, Sherman ME. Comparison of Age Distribution Patterns for Different Histopathologic Types of Breast
Carcinoma. Cancer Epidemiol Biomarkers Prev 2006:15:1899-905. doi:10.1158/1055-9965.EPI-06-0191. 